DOI: https://dx.doi.org/10.4314/sinet.v44i1.7

\title{
Depression, Anxiety and Associated factors among COVID-19 Patients at Armed Force Comprehensive COVID-19 Center Hospital in Ethiopia
}

\author{
Asnake Fantu1, Abdissa Boka ${ }^{2, *}$ and Negalign Getahun² \\ 1Department of Psychiatry, College of Health Science, Defense University, Addis Ababa, Ethiopia \\ ${ }^{2}$ School of Nursing \& Midwifery, College of Health Sciences, Addis Ababa University, Addis \\ Ababa, Ethiopia. E-mail: bokaabdisa@yahoo.com
}

\begin{abstract}
The coronavirus disease 2019 (COVID-19) pandemic has a significant impact on the public mental problem. The anxiety and depression symptoms are a common emotional reaction to the COVID19 pandemic. The objective of the study was to assess the level of depression and anxiety reactions and associated factors among COVID-19 patients at the COVID-19 center of the Armed Force Comprehensive Hospital in Ethiopia. Across-sectional study was conducted among 150 COVID-19 patients who were interviewed for the survey in the Afaan Oromo language. The Beck's Depression Inventory and Beck's Anxiety Inventory measuring scale questionnaires were adapted and used to measure depression and anxiety of COVID-19 patients. Data were analyzed by logistic regression using SPSS computer software version 23. Mean age of the COVID-19 patients was 29 with a standard deviation of \pm 7.6 years. Among the total respondents, $92(61.3 \%)$ were males. The overall prevalence of depression from borderline clinical to severe was $84 \%$ and anxiety from moderate to severe was $86.7 \%$. The multivariate regression analysis showed that being married, divorced, fear of death, poor sleep quality, fear of re-infection, less family support, loneliness, and lower oxygen saturation were positively associated with depression and anxiety reactions. From study participants, the majority of them were presented with features of depression and anxiety. Being married, divorced, fear of death, poor sleep quality, fear of re-infection, less family support, loneliness, and lower oxygen saturation were the associated factors with depression and anxiety reaction for COVID-19 patients.
\end{abstract}

\section{Keywords: Anxiety, COVID-19 center, COVID-19 patients, Depression, Ethiopia}

\section{INTRODUCTION}

Coronavirus disease 2019 (COVID-19) is first recognized in Wuhan, Hubei Province of China since December 2019, which is a worldwide pandemic of public health emergency and has features spreading rapidly around the globe (Chinazzi et al., 2020; Nishiura et al., 2020; Zhou et al., 2020). By August 31, 2020, the World Health Organization (WHO) reported that more than 41 million were infected 1,130,220 deathsglobally, $1,675,580$ infections and 40,309 deaths in African, and 90,490 cases and 1,371 deaths in Ethiopia which is increasing from day to day (WHO, 2020). Anxiety and depression are common mental illnesses in the world and the most impact causing mental problems. During this outbreak, the mental health issue is not an ignored problem since there is the influence of the outbreak on the mental health problem. As different studies revealed that depression and anxiety are commonly persistent among chronic diseases. Symptoms of depression and anxiety are common psychological reactions to
COVID-19 (Dai et al., 2020; Hopwood \& Stephens, 2000; Natale et al., 2019; Pathan \& Thakur, 2020; Polikandrioti et al., 2015; WHO, 2017).

As different studies indicated that among COVID-19 patients surveyed, a significant proportion was experiencing mental illnesses; mainly depression and anxiety that cause trouble of symptom regulator and have decreased quality of life. Though recently published researches on the psychological impact of COVID-19 are mainly focused on the healthcare workers despite, the general public who were worried about the risks of infection and protective measures, resulting in psychological distress (Dai, Hu, Xiong, Qiu, \& Yuan, 2020; Wang et al., 2020; Zhu et al., 2020).

Even though COVID-19 is a new strain of coronaviruses, it is known by causing diseases ranging from cold to more severe illnesses like Middle East Respiratory Syndrome (MERS) and Severe Acute Respiratory Syndrome (SARS). Severe cases of this disease can lead to cardiac problems like heart failure, and respiratory syndrome, and

*Author to whom correspondence should be addressed. 
renal failure as well as death. Anxiety is one of the common psychosocial problems that affect the majority of populations. Many of the current research evidence suggests that isolated and quarantined people were experiencing a significant level of anxiety, stress, and confusion (Ashour, Elkhatib, Rahman, \& Elshabrawy, 2020; Brooks et al., 2020; Holshue et al., 2020).

As different strategies were implemented by different countries in the world to minimize the risk of the COVID-19 transmission in the community. Ethiopia also declared an emergency state to minimize the risk of COVID-19 transmission by applying travel restrictions, physical distancing, quarantine, frequent handwashing, reducing the capacity of public transportation, and stay-at-home preventive and control strategies. Despite many efforts to reduce the risk of COVID-19 transmission, mental health problems and psychological impact among COVID-19 patients have remained high. Therefore, this study aimed to assess the level of depression, anxiety, and associated factors among COVID-19 patients in the COVID-19 center of an Armed Force Comprehensive Hospital in Ethiopia.

\section{METHODS AND MATERIALS}

\section{Study Design, Setting, and Population}

The institution-based cross-sectional study was conducted from 15 July to 30 August 2020 at COVID-19 Center of Armed Force Comprehensive Hospital in Ethiopia. The COVID-19 center of Armed Force Comprehensive Hospital is found in Oromia regional state in Bishoftu town which is about 40 kilometers far from Addis Ababa (the capital city of Ethiopia) to the Northeast. The hospital gives service for 600 SARS Cov-2 positive patients. All SARS Cov-2 positive (18 years or older) and admitted patients were included, except those who were critically ill and unable to talk at the time of data collection. Samples of 150 COVID-19 patients were selected by simple random sampling techniques.

The data were collected by three nurses holding a Bachelor of Science degree under the supervision of a senior psychiatry professional. The data collectors were provided one day of concentrated training on the content of the measuring tool and how to select a participant for the interview. The principal investigators also made a continuous follow-up to ensure the quality of the data collected. The Institutional Review Board of the College of Health Sciences at Defense University had reviewed and approved the research project and a letter of cooperation was written to COVID-19 Center of Armed Force Comprehensive Hospital. After permission was obtained from the authorities of the study setting, the written informed consents were secured from each participant and the study was conducted as per the Declaration of Helsinki.

\section{Measurements}

The socio-demographic characteristics and other factors for COVID-19 patients were recorded using 14-items. These include sex, age, religion, educational status, occupation, marital status, fear of death, stigma, absence of sleep, fear of reinfection, less social support, loneliness, economic impact, and lower oxygen saturation. The measurements for depression and anxiety concerning COVID-19 positive patients were recorded using 21-items Beck Depression Inventory and 21-items Beck Anxiety Inventory.

Both the Beck Depression Inventory (BDI) and Beck Anxiety Inventory (BAI) were used to measure depression and anxiety reactions of the COVID-19 patients, respectively. Both tools contain 21 items with a Likert scale ranging from 0 (not at all) to 3 (severely). The total score (0-63) was obtained by summing up the scores of all items. The cut-off point for levels of depression interpretation was $\geq 17$ score points without focusing on normal mood (1-10) and mild mood disturbance (11-16) scores. Borderline clinical depression (17-20), moderate depression (21-30), severe depression (31-40), and extreme depression (>40) scores were considered for the level of depression assessment. Again, for the level of anxiety interpretation, the cut-off point for individuals with anxiety problems was $\geq 22$-point scores without emphasizing a mild form of anxiety reaction because it is the usual problem without causing significant clinical distress, occupational, and functional impairment. The moderate form of 
anxiety with a grand sum between 22-35 score and the severe form of anxiety with a grand sum of $\geq$ 36 scores was considered potentially concerning levels of anxiety for assessment (Beck, Epstein, Brown, Steer, \& psychology, 1988; Sprinkle et al., 2002).

Data was collected in the Afaan Oromo language using a face-to-face interviewer. In this regard, the questionnaire was translated from English to the Afaan Oromo language by a fluent translator and then back-translated to English by another fluent translator. Before data collection, we assessed the Afaan Oromo language version of the BDI and BAI to ensure meaning equivalence with the original English.

\section{Data Processing and Analysis}

Data were coded, entered, and cleaned using Epi-data version 3.1 and then transferred to SPSS version 23.0 for analysis. Frequency distributions were computed for sociodemographic variables and the patient's reactions. The independent variables in this study were sociodemographic factors and the dependent variables were depression and anxiety. The independent variables associations with depression and anxiety reactions were analyzed first by using bivariate analysis. Then, only those variables with p-values $\leq 0.25$ were taken as a candidate for multivariate logistic analysis. In the multivariate logistic analysis pvalue $<0.05$ was considered to be statistically significant.

\section{RESULTS}

\section{Sociodemographic Characteristics of the Participants}

All of the study participants (150) were interviewed with a $100 \%$ response rate. The age of respondents ranges from 18 to 60 years with the mean age of $29 \pm 7.6$ years. Among the total respondents, 92 (61.3\%) were males, 77 (51.3\%) were married and $71(47.3 \%)$ were Christian by religion. Half $(50 \%)$ of them have attended College/University education and $76(50.6 \%)$ were military members(Table 1).
Table 1. Distribution of participants by sociodemographic characteristics of COVID-19 patients at Armed Force Comprehensive Hospital in Oromia, Ethiopia $(n=150)$.

\begin{tabular}{llll}
\hline Characteristics & Category & $\begin{array}{l}\text { Frequency } \\
\text { (n) }\end{array}$ & $\begin{array}{l}\text { Percentage } \\
(\%)\end{array}$ \\
\hline Sex & Male & 92 & 61.3 \\
Age & Female & 58 & 38.7 \\
& $18-22$ & 11 & 7.3 \\
& $23-27$ & 40 & 26.7 \\
\multirow{5}{*}{ Religion } & $28-32$ & 53 & 35.3 \\
& $\geq 33$ & 16 & 10.7 \\
& Orthodox & 71 & 47.3 \\
\multirow{5}{*}{ Educational } & Muslim & 29 & 19.3 \\
\multirow{4}{*}{ Occupation } & Protestant & 42 & 28 \\
& Others & 8 & 5.3 \\
& Primary school & 35 & 23.3 \\
& High school & 40 & 26.7 \\
Marital status & College/University & 75 & 50 \\
& Jobless & 5 & 3.3 \\
& Civilian workers & 69 & 46 \\
& Military & 76 & 50.7 \\
& Single & 53 & 35.3 \\
& Married & 77 & 71.3 \\
& Divorce & 20 & 13.3 \\
\hline
\end{tabular}

Factors that contribute to the presence of anxiety and depression among COVID-19 patients at the Armed Force Comprehensive COVID-19 center Hospital.

Among the factors that contribute to depression and anxiety for COVID-19 patients, fear of death $(96.7 \%)$, lower oxygen saturation $(93.3 \%)$, loneliness $(91.3 \%)$, fear of re-infection $(85.3 \%)$, economical problem (80 \%), and stigma (66.7\%) (Table 2).

Table 2. Factors that contribute to the presence of anxiety and depression among COVID-19 patients at Armed Force Comprehensive Hospital in Oromia, Ethiopia $(n=150)$.

\begin{tabular}{llll}
\hline Factors & Yes/No & $\begin{array}{l}\text { Frequency } \\
(\mathrm{n})\end{array}$ & $\begin{array}{l}\text { Percentage } \\
(\%)\end{array}$ \\
\hline Fear of death & Yes & 145 & 96.7 \\
Stigma & No & 5 & 3.3 \\
& Yes & 100 & 66.7 \\
Poor sleep quality & No & 50 & 33.3 \\
& Yes & 126 & 84 \\
Fear of re-infection & No & 24 & 16 \\
& Yes & 128 & 85.3 \\
Less family support & Yo & 22 & 14.7 \\
& No & 104 & 71.3 \\
Loneliness & Yes & 137 & 30.7 \\
\multirow{2}{*}{ Economic problem } & No & 13 & 91.3 \\
& Yes & 120 & 8.7 \\
Lower & No & 30 & 80 \\
saturation & Yes & 140 & 20 \\
\hline
\end{tabular}


The Prevalence of Depression and Anxiety among COVID-19 patients

According to the multidimensional scoring system of Beck's depression and anxiety Inventory measuring scales, the proportion of mild, borderline clinical, moderate, and severe depression was $7.3 \%, 18.7 \%, 56 \%$, and $9.3 \%$, respectively. Similarly, mild, moderate, and severe anxiety was $6 \%, 10 \%$, and $76.7 \%$, respectively. The overall prevalence of depression from borderline clinical to severe was $84 \%$ while that of anxiety from moderate to severe was $86.7 \%$.

\section{Factors Associated with Depression and Anxiety of the COVID-19 patients}

In the bivariate analyses, sociodemographic factors (age, sex, and marital status), and factors for depression and anxiety level concerning COVID19 patients (fear of death, stigma, fear of reinfection, loneliness, less family support, poor sleep quality, low oxygen saturation, and economic problem) had a statistically significant association with depression and anxiety level, while religion, educational status, and occupation did not have an association with depression and anxiety. However, in the multivariate logistic regression analysis, age, marital status, fear of death, fear of re-infection, loneliness, less family support, poor sleep quality, low oxygen saturation, and an economic problem had a statistically significant association with both depression and anxiety.

Those who were married and divorced were more likely develop depression and anxiety than others ( $\mathrm{AOR}=3.61$; $\mathrm{CI}$ : 3.12, 3.74), $\mathrm{AOR}=2.60$; $\mathrm{CI}: 1.56$, $4.36)$, $(\mathrm{AOR}=3.12$; $\mathrm{CI}: 0.54,3.34)$, and $(\mathrm{AOR}=1.60$; $\mathrm{CI}$ : $1.64,3.68)$, respectively. Fear of death among depressed and anxious patients was $(\mathrm{AOR}=2.26$; $\mathrm{CI}$ : $1.30,4.67)$ and (AOR $=4.18$; CI: $1.32,5.33$ ). Poor sleep quality was more likely affects depressed and anxious COVID-19 patients were (AOR $=3.56$; CI: $1.50,5.44)$ and (AOR $=2.12$; CI: $1.42,3.59)$. Fear of reinfection, less family support, loneliness and lower oxygen saturation among depressed and anxious patients were (AOR $=3.61$; CI: 2.21,5.87), $(\mathrm{AOR}=2.17$; CI: $1.42,3.38),(\mathrm{AOR}=2.43$; CI: $1.34,5.91),(\mathrm{AOR}=3.11$; CI: 1.32, 3.35), (AOR $=4.01$; CI: $2.33,8.77),(\mathrm{AOR}=3.18$; CI: 1.42, 3.39), (AOR =2.22; CI: 1.43,4.54), (AOR =4.10; CI: $1.42,5.35)$, respectively positively associated (Table 3).

Table 3. Factors Associated with Depression and Anxiety among COVID-19 patients at CoviD-19 center of Armed Force Comprehensive Hospital Oromia, Ethiopia $(\mathbf{n}=150)$.

\begin{tabular}{llllll}
\hline Explanatory Variables & & \multicolumn{2}{c}{ Depression } & \multicolumn{2}{c}{ Anxiety } \\
\cline { 3 - 6 } & & COR, 95\%CI & AOR,95\%CI & COR,95\%CI & AOR,95\%CI \\
\hline Age & $18-22$ & 1.00 & 1.00 & 1.00 & 1.00 \\
& $23-27$ & $2.26(1.30,4.67)$ & $2.19(1.42,3.39)$ & $3.15(0.55,5.11)$ & $2.11(.76,3.66)$ \\
& $28-32$ & $2.85(0.69,4.23)$ & $3.14(0.54,3.34)$ & $2.26(1.30,4.67)$ & $1.19(1.12,3.30)^{*}$ \\
Sex & $\geq 33$ & $3.43(0.54,4.33)$ & $4.72(0.34,5.76)$ & $2.61(2.13,3.14)$ & $2.11(1.35,3.77)^{*}$ \\
Marital status & Male & 1.00 & 1.00 & 1.00 & 1.00 \\
& Female & $8.11(1.94,13.93)$ & $2.84(1.12,6.78)$ & $2.24(1.42,4.52)$ & $2.35(1.14,4.51)^{*}$ \\
& Single & 1.00 & 1.00 & 1.00 & 1.00 \\
Fear of death & Married & $0.43(0.41,0.87)$ & $3.61(3.12,3.74)$ & $0.76(0.56,1.92)$ & $2.60(1.56,4.36)^{*}$ \\
& Divorce & $2.85(0.69,4.25)$ & $3.12(0.54,3.34)$ & $1.67(1.32,2.52)$ & $1.60(1.64,3.68)^{*}$ \\
Poor sleep quality & Yes & $5.26(1.50,9.76)$ & $2.26(1.30,4.67)$ & $2.08(1.02,4.24)$ & $4.18(1.32,5.33)^{*}$ \\
& No & 1.00 & 1.00 & 1.00 & 1.00 \\
Fear of re-infected & Yes & $0.66(0.01,5.67)$ & $3.56(1.50,5.44)$ & $1.15(1.12,2.69)$ & $2.12(1.42,3.59)^{*}$ \\
& No & 1.00 & 1.00 & 1.00 & 1.00 \\
Less family support & Yes & $1.43(1.22,7.23)$ & $3.61(2.21,5.87)$ & $1.43(0.62,3.13)$ & $2.17(1.42,3.38)^{*}$ \\
& No & 1.00 & 1.00 & 1.00 & 1.00 \\
Loneliness & Yes & $1.11(1.01,3.54)$ & $2.43(1.34,5.91)$ & $1.23(0.76,3.25)$ & $3.11(1.32,3.35)^{*}$ \\
& No & 1.00 & 1.00 & 1.00 & 1.00 \\
Economic problem & Yes & $4.17(0.26,8.12)$ & $4.01(2.33,8.77)$ & $2.21(0.41,4.46)$ & $3.18(1.42,3.39)^{*}$ \\
& No & 1.00 & 1.00 & 1.00 & 1.00 \\
Lower oxygen saturation & Yes & $2.22(1.82,3.28)$ & $1.92(1.68,3.78)$ & $1.52(0.53,2.44)$ & $2.13(1.43,3.38)^{*}$ \\
& No & 1.00 & 1.00 & 1.00 & 1.00 \\
& Yes & $6.31(3.33,12.32)$ & $2.22(1.43,4.54)$ & $1.57(0.89,4.13)$ & $4.10(1.42,5.35) *$ \\
\hline
\end{tabular}

${ }^{*}$ Refers to a significant association ( $p$-value $<0.05$ )

$\mathbf{1 . 0 0}=$ reference

AOR: Adjusted odds ratio

COR: Crudes odds ratio

CI: confidence interval 


\section{DISCUSSION}

In this study, we assessed the prevalence of depression and anxiety and their associated factors among COVID-19 patients at the Armed Force Comprehensive COVID-19 center Hospital in Oromia, Ethiopia. The age of respondents ranges from 18 to 60 years with the mean age of $29 \pm 7.6$ years. Of the total participants, $84 \%$ had depression and $86.7 \%$ had anxiety. This high prevalence of depression and anxiety at the COVID19 center can exert an impact on the patient's life or prognosis. Due attention is necessary because global health threats with devastating consequences of COVID-19 and the burden of depression and anxiety can potentially impact the outcome of this pandemic disease (Chinazzi et al., 2020; Nishiura et al., 2020; Zhou et al., 2020).

The prevalence of depression and anxiety were higher (84\% depression and $86.7 \%$ anxiety) than the findings of the studies done in Huoshenshan Hospital, Wuhan China, (28.47\% and 34.72\%) and Jianghan Fangcang Shelter Hospital in Wuhan China $13.4 \%$ and $8.6 \%$, respectively (Dai et al., 2020; Kong et al., 2020; Tariku, 2020). This might be because that study had been conducted in a different community with different socio-economic factors.

Another finding of our present study identified that older age, being female, married, and divorced have a positive association with depression and anxiety which was similar to the study conducted in China, Italy, and Spain (Barzilay et al., 2020; Dai et al., 2020; GonzálezSanguino et al., 2020; Kong et al., 2020; Rossi et al., 2020; Sher, 2020; Wang et al., 2020).

The result found in this study indicated that those respondents who had been fear of death were 2 times more likely to have depression and 4 times more likely to have anxiety compared to those who had not been with fear of death. This finding was similar to the result of other studies (Barzilay et al., 2020; González-Sanguino et al., 2020). This might be partly explained by the fact that fear of death causes a multidimensional effect on the psychology of respondents while they think more about the quarantine, loss of social support, and decreased family relationships.

Those who had been known to be less family support were 2 times more likely to have depression and 3 times more likely to have anxiety compared to those who have good family support.
On the other hand, even if it was not associated with both depression and anxiety, stigma might have a psychological scar that might be painful. Those who had poor sleep quality and fear of reinfection had more likely 3 times depression and 2 times anxiety compared to those who had no sleep problem and fear of re-infection. This study substantiates with the previous study conducted in other settings (Chang et al.; Dai et al., 2020; Sher, 2020). The economic problem of the respondents, in general, had the possibility of altering the presence of depression and anxiety which corroborates with a previous study (Barzilay et al., 2020; Brooks et al., 2020; Sher, 2020). Another finding from the present study was loneliness and low oxygen saturation of COVID-19 patients who were more likely to have depression and anxiety symptoms. COVID-19 had a significant impact on people's health, economy, sleep, fear, and relationship (Barzilay et al., 2020; Brooks et al., 2020; Dai et al., 2020; Endomba, Wouna, \& Danwang, 2020; González-Sanguino et al., 2020; Guo et al., 2020; Hirvonen, Abate, \& de Brauw, 2020; Huang \& Zhao, 2020; Ma et al., 2020) our study of why the prevalence of depression and anxiety is high among COVID-19 patients. This is the first study conducted in Ethiopia to assess depression and anxiety at the COVID-19 center of Armed Force Comprehensive Hospital in Ethiopia. However, the study design itself has limitations since it is not identifying the causal factor relationship.

\section{CONCLUSIONS}

There is a high reaction feeling of depression and anxiety among COVID-19 patients. Factors associated with depression and anxiety among COVID-19 patients were fear of death, loneliness, lower oxygen saturation, and lack of social support. Efforts need to be focused to raise awareness of how depression and anxiety exert an impact on the COVID-19 patients.

\section{ACKNOWLEDGMENTS}

We acknowledge the Defense University of Ethiopia for the provision of financial support to this research project. Also, we acknowledged data collectors, supervisors, and study participants for their valuable contributions. 


\section{REFERENCES}

1. Ashour., H., Elkhatib., M., Walid., F., Rahman, M., \& Elshabrawy, H. (2020). Insights into the recent 2019 novel coronavirus (SARS-CoV-2) in light of past human coronavirus outbreaks. Pathogens 9:186.

2. Barzilay, R., Moore, T., Greenberg, D., DiDomenico, G., Brown, L., White, L., Raquel, E. (2020). Resilience, COVID-19-related stress, anxiety and depression during the pandemic in a large population enriched for healthcare providers. Translational psychiatry 10: 1-8.

3. Beck, A., Epstein, N., Brown, G., Steer, R., psychology, C (1988). An inventory for measuring clinical anxiety: psychometric properties. Journal of consulting E psychology clinical 56: 893.

4. Brooks, S., Webster, R., Smith, L., Woodland, L., Wessely, S., Greenberg, N., Rubin, G. (2020). The psychological impact of quarantine and how to reduce it: rapid review of the evidence. The Lancet 95:912-920.

5. Chang, K., Hou, W., Pakpour, A., Lin, C., Griffiths, M., $\mathrm{Hu}$, Y., Chen, Y., Zheng, Y., You, C., Tan, J., Hu, L., Zhang, Z., Ding, L., Guo, Q., Zheng, Y., Shi, J., Wang, J., Li, G., Li, C., Fromson, J.A., Xu, Y., Liu, X., Xu, H., Zhang, T., Lu, Y., Chen, X., Hu, H., Tang, Y., Yang, S., Zhou, H., Wang, X., Chen, H., Wang, Z., Yang, Z. (2020). Psychometric Testing of Three COVID-19-Related Scales Among People with Mental Illness. International Journal of Mental Health and Addiction 18: 1-13.

6. Chinazzi, M., Davis, J., Ajelli, M., Gioannini, C., Litvinova, M., Merler, S., Piontti, A., Mu, K., Rossi, L., Sun, K. (2020). The effect of travel restrictions on the spread of the 2019 novel coronavirus (COVID-19) outbreak. Science 368:395-400.

7. Dai, L.., Wang, X., Jiang, T., Li, P., Wang, Y., Wu, S.., Jia, L., Liu, M., An, L., Cheng, Z. (2020). Anxiety and depressive symptoms among COVID-19 patients in Jianghan Fangcang Shelter Hospital in Wuhan, China. PloS one, 15(8), e0238416.

8. Dai, Y., Hu, G., Xiong, H., Qiu, H., Yuan, X. (2020). Psychological impact of the coronavirus disease 2019 (COVID-19) outbreak on healthcare workers in China. MedRxiv.doi:https://doi.org /10.1101 /2020.03.03.20030874

9. Endomba, F., Wouna, D., Danwang, C. (2020). Mental health during the coronavirus disease 2019 (Covid-19) pandemic: more is still to be done. The Pan African Medical Journal 35:58-65.
10. González-Sanguino, C., Ausín, B., ÁngelCastellanos, M., Saiz, J., López-Gómez, A., Ugidos, C., Muñoz, M. (2020). Mental health consequences during the initial stage of the 2020 Coronavirus pandemic (COVID-19) in Spain. Brain, Behavior and Immunity 87:172-6.

11. Guo, Q., Zheng, Y., Shi, J., Wang, J., Li, G., Li, C., Fromson, J.A., Xu, Y., Liu, X., Xu, H.J.B. (2020). Immediate psycho-logical distress in quarantined patients with COVID-19 and its association with peripheral inflammation: a mixed-method study. Brain, Behavior, and Immunity 88:17-27.

12. Hirvonen, K., Gashaw Tadesse, de Brauw, A. (2020). Food and nutrition security in Addis Ababa, Ethiopia during COVID-19 pandemic: ESSP Working Paper 143. Washington DC: IFPRI.

13. Holshue, M.., DeBolt, C., Lindquist, S., Lofy, K.., Wiesman, J., Bruce, H., Spitters, C., Ericson, K., Wilkerson, S., Tural, A. (2020). First case of 2019 novel coronavirus in the United States. New England Journal of Medicine;382:929-936.

14. Hopwood, P., Stephens, R. (2000). Depression in patients with lung cancer: prevalence and risk factors derived from quality-of-life data. Journal of clinical oncology 18(4):893-893.

15. Huang, Y., Zhao, N. (2020). Generalized anxiety disorder, depressive symptoms and sleep quality during COVID-19 outbreak in China: a webbased cross-sectional survey. Psychiatry Res.288:112954.

16. Kong, X., Zheng, K., Tang, M., Kong, F., Zhou, J., Diao, L., Wu, S., Jiao, P., Su, T., Dong, Y. (2020). Prevalence and factors associated with depression and anxiety of hospitalized patients with COVID-19. MedRxiv doi:https: //doi.org/10.1101/2020.03.24.20043075.

17. Ma, Y., Li, W., Deng, H.., Wang, L., Wang, Y., Wang, P.., Bo, H.., Cao, J., Wang, Y., Zhu, L. (2020). Prevalence of depression and its association with quality of life in clinically stable patients with COVID-19. Journal of affective disorder 275: 145148.

18. Natale, P., Palmer, S., Ruospo, M., Saglimbene, V., Rabindranath, K., Strippoli, G. (2019). Psychosocial interventions for preventing and treating depression in dialysis patients. Cochrane database of systematic reviews. DOI:10.1002/14651858.CD004542.pub3.

19. Nishiura, H., Jung, S., Linton, N., Kinoshita, R., Yang, Y., Hayashi, K., Kobayashi, T., Yuan, B., Akhmetzhanov, A. (2020). The extent of transmission of novel coronavirus in Wuhan, 
China, 2020: Multidisciplinary Digital Publishing Institute. 9: 330 .

20. Pathan, A., Thakur, S. (2020). Economic Analysis of Legislative Process of Parliament in the Light of Pandemic of Covid-19 and India: Revisiting Existing Physical Infrastructure from the Prism of Evolving Information and Communication Technology (ICT). Journal of Critical Review 7:6493-6504.

21. Polikandrioti, M., Goudevenos, J., Michalis, L., Koutelekos, J., Kyristi, H., Tzialas, D., Elisaf, M. (2015). Factors associated with depression and anxiety of hospitalized patients with heart failure. Hellenic J. Cardiol 56: 26-35.

22. Rossi, R., Socci, V., Talevi, D., Mensi, S., Niolu, C., Pacitti, F., Di Marco, A., Rossi, A., Siracusano, A., Di Lorenzo, G. (2020). COVID-19 pandemic and lockdown measures impact on mental health among the general population in Italy. Front Psychiatry 11:790.

23. Sher, L.J.S.M. (2020). COVID-19, anxiety, sleep disturbances and suicide. Sleep Medicine. 70:124

24. Sprinkle, S., Lurie, D., Insko, S., Atkinson, G., Jones, G., Logan, A., Bissada, N. (2002). Criterion validity, severity cut scores, and test-retest reliability of the Beck Depression Inventory-II in a university counseling center sample. Journal of Counseling psychology 49: 381.

25. Tariku Mandaras (2020). Corona virus disease (COVID-19) and Mental Health in the
Community. East African Journal of Health Biomedical Sciences. 4: 1-4.

26. Wang, C., Pan, R., Wan, X., Tan, Y., Xu, L., Ho, C.S., Ho, R. (2020). Immediate psychological responses and associated factors during the initial stage of the 2019 coronavirus disease (COVID-19) epidemic among the general population in China. International journal of environmental researchpublic health. 17: 1729

27. World Health Organization. (2017). Other common mental disorders: global health estimates. Geneva: World Health Organization, 1-24. Found at URL: https://www.who.int/mental_health/managem ent/depression/prevalence_global_health_estim ates/en/

28. World Health Organization. (2020). Coronavirus disease 2019 (COVID-19): situation report, 82. Found at URL:https://apps.who.int/iris/ handle/10665/331780

29. Zhou, F., Yu, T., Du, R., Fan, G., Liu, Y., Liu, Z., Xiang, J., Wang, Y., Song, B., Gu, X. (2020). Clinical course and risk factors for mortality of adult inpatients with COVID-19 in Wuhan, China: a retrospective cohort study. The lancet. Doi:https://doi.org /10.1016/s0140-6736(20) 30566-3.

30. Zhu, Z., Xu, S., Wang, H., Liu, Z., Wu, J., Li, G., Miao, J., Zhang, C., Yang, Y., Sun, W. (2020). COVID-19 in Wuhan: Immediate Psychological Impact on 5062 Health Workers. The Lancet. DOI:10.1016/jeclinm100443. 